\title{
PRELIMINARY RESULTS ON THE APPARENT SIZE OF THE SOURCES OF TYPE III BURSTS OBSERVED AT LOW FREQUENCIES
}

\author{
HECTOR ALVAREZ* \\ Radio Astronomy Observatory, \\ The University of Michigan, \\ Ann Arbor, Mich., U.S.A.
}

\begin{abstract}
We present preliminary results on the apparent angular size of the sources of four type III bursts observed between 3500 and $50 \mathrm{kHz}$ from the IMP- 6 spacecraft. The observations were made with a dipole rotating in the plane of the ecliptic where the sources are assumed to be. The apparent angular sizes obtained are unexpectedly large. We discuss different explanations for the results. It seems that the scattering of radio waves by electron density inhomogeneities is the most likely cause.

We report a temporal increase of the apparent angular size of the source during the burst lifetime for some bursts. From its characteristics it apears to be a real effect.
\end{abstract}

\section{Introduction}

Type III solar bursts are produced by a stream of fast particles ejected by the Sun. This stream excites plasma waves in the ambient corona that couple into electromagnetic waves at the fundamental and/or second harmonic of the local plasma frequency.

During the past few years considerable knowledge has been obtained about these bursts (see reviews by different authors in Space Sci. Rev. 16, 1974). In particular, low frequency observations from spacecraft have given information about the identity of the particles, the trajectory of the stream, the propagation of the particles, the mode of emission, etc.... The theoretical aspects of emission mechanisms have received increasing attention, as it is evidenced by many papers in this issue.

The size of the source is an important characteristic of type III bursts about which little is known. This parameter is difficult to determine from radio astronomical observations because of the smearing effect of radio wave scattering in the interplanetary medium (see for example Steinberg, 1972; Riddle, 1974). At best the observations can give an apparent size. Measurements made at meter wavelength with ground based instruments indicate that the angular size increases with decreasing frequency (Wild and Sheridan, 1958; Goldstein, 1959; Weiss and Sheridan, 1962; Elgaroy and Rodberg, 1963). Since the exciter particles are guided by the interplanetary magnetic field, it is expected that velocity dispersion should increase the length of the stream while the opening of the field lines should increase the lateral size. Particle scattering should also increase the actual source size.

The experiment that the University of Michigan had aboard the IMP-6 satellite gives information about the apparent size of type III sources provided that the altitude of the source of the plane of the ecliptic is known. The measurements

\footnotetext{
* Permanent address: Centro de Investigación de Astronomía, Apartado 264, Mérida, Venezuela.
} 
were made at frequencies between 900 and $50 \mathrm{kHz}$ corresponding to heliocentric distances of about 10 to 215 solar radii, $R_{\odot}$. Assuming that the trajectory of the stream centroid is on the plane of the ecliptic we find that the apparent angular size increases to unexpectedly large values with decreasing frequency. We discuss the effects that the assumptions made and radio wave scattering may have on the results.

\section{The Experiment}

The experiment consists of an eight-channel receiver connected to a $55-\mathrm{m}$ tip-to-tip dipole. The receiver steps continuously through the eight channels completing a cycle in about $4.2 \mathrm{~s}$. The channels are centered at 3500, 900,600, $350,230,130,80$ and $50 \mathrm{kHz}$, with $10 \mathrm{kHz}$ bandwidth. The dipole rotates with a period of about $11 \mathrm{~s}$ in a plane that is within $2^{\circ}$ of the plane of the ecliptic.

At the observing frequencies, except $3500 \mathrm{kHz}$, the antenna is a short dipole with a $\cos ^{2}$ power pattern. A radio source would then be observed at the antenna terminals as a sinusoidally amplitude modulated signal. The depth of the modulation depends on two parameters, the size of the source and the altitude of the source above the dipole rotation plane. One of them can be determined only when the other is known. A given amplitude modulated pattern can be produced by a source of a certain size located on the rotation plane or by a smaller source off the rotation plane.

There are uncertainties in our measurement of small signals and also of large signals that drive the receiver to saturation. This is due to a combination of the nonlinear response of the receiver at those levels and of the discreteness of the telemetry steps. For the events studied here we had problems only with the small signals.

\section{Data}

At each frequency the time profile of a burst is divided into subintervals of specified length. A computer program assumes a sinusoidal modulation of known period and performs an independent least-squares fit to the observations in each subinterval to determine the modulation which best fits.

The amount of modulation is measured by the modulation parameter $C$ :

$$
C=\frac{1}{2} \frac{S_{M}-S_{m}}{S_{M}}
$$

where $S_{M}$ and $S_{m}$ are the amplitudes of the peak and trough, respectively, of the modulated signal. $C$ varies between 0 and $\frac{1}{2}$. A computer plots demodulated burst profiles (amplitude versus time), and also the $C$ value for each subinterval.

Because of the uncertainties in the measurement of a deep trough, values of $C \geqslant 0.49$ are not reliable. This applies particularly to the $3500 \mathrm{kHz}$ observations; however, observations at this frequency were not used because the antenna is not 
a short dipole. Due to the uncertainties in measuring $S_{M}-S_{m}$ when $S_{M} \approx S_{m}$ we assumed that values of $C \leqslant 0.1$ were equal to zero.

The four events analyzed occurred on 16 May, 1971;28 February, 1972; 27 March, 1972 and 19 August 1972. We have chosen them because we believe that they are the ones whose parameters we know best at the present time (Alvarez et al. 1975). The radio bursts were associated with fast solar electrons observed at the Earth and therefore their trajectories passed close to the Earth. The radiation was second harmonic and the electron density distributions were such that the $50 \mathrm{kHz}$ bursts occurred at or beyond the Earth's orbit. This is consistent with the fact that no modulation was observed at that frequency. The 16 May, 1971 event will be used to illustrate the results. The findings for the other three events are similar.

\section{Analysis}

The analysis is based on the comparison of the observations with the model illustrated in Figure 1. There we see a cross section of the antenna pattern, with the antenna axis in the plane of the figure. The model assumes that the source is a uniformly illuminated spherical cap of circular contour. The source defines then a conical solid angle with angular diameter $D$. The axis of the cone makes an angle $\alpha$ with the antenna axis. For any source trajectory that starts from the Sun there is in general a different $\alpha$ for each frequency. The only case where the $\alpha$ 's are equal is when $\alpha=0$, that is, when the sources are contained in the plane of rotation of the dipole (ecliptic). We have computed $C$ as a function of $D$ for different $\alpha$. Figure 2 shows some of the curves.

The plots of measured $C$ versus time show some systematic variations of $C$ within the burst life time. After the burst becomes strong the modulation parameter shows a monotonic decrease that will be discussed later. To compute

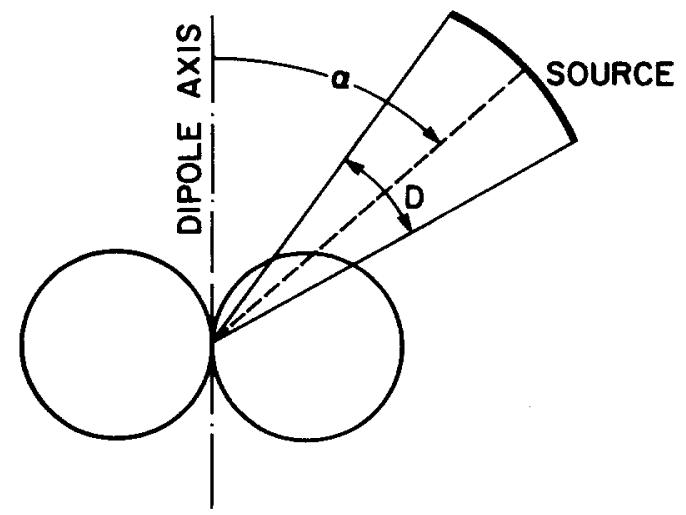

Fig. 1. Model geometry. The antenna pattern is that of a short dipole. The direction to the source center makes an angle $\alpha$ with the antenna axis. The source is assumed to be a uniformly illuminated spherical cap subtending an angular diameter $D$. 


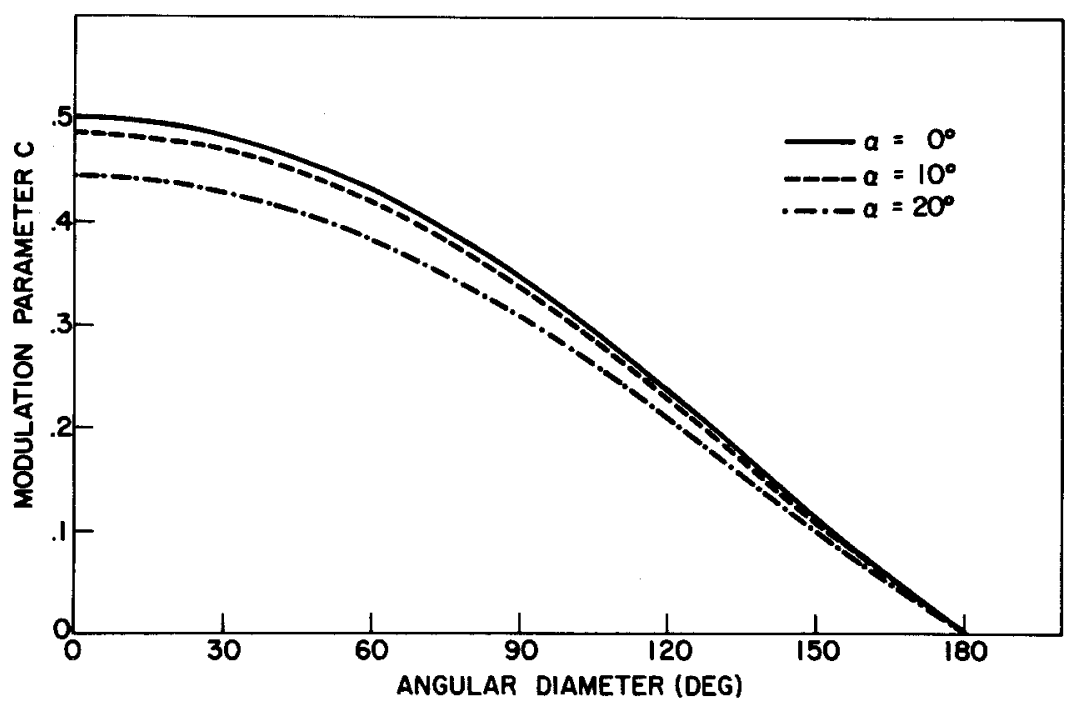

Fig. 2. Modulation parameter $C$ as a function of the source angular diameter for different source elevations $\alpha$.

an average apparent angular diameter of the source at each frequency we selected the C's that do not depart significantly from the mean.

Figure 3 shows a plot of average apparent angular diameter versus frequency for $\alpha=0$. The choice of $\alpha=0$ is discussed later. The points at $D=180^{\circ}$ were not observed and represent the value expected when the source is at the Earth and radiates at the second harmonic. The plasma frequency at $1 \mathrm{AU}$ was obtained from the study of Alvarez et al. (1975). Using the electron density distribution determined by these authors we plotted apparent angular diameter versus heliocentric distance $r$, in solar radii, $R_{\odot}$. This is shown in Figure 4 . The circle represents the prediction at $1 \mathrm{AU}\left(1 \mathrm{AU}=215 R_{\odot}\right)$. Figure 5 shows the position of the sources determined by Alvarez et al. (1975). The solid circles represent the plasma levels where the second harmonic bursts originated. The angles at which the broken line arcs are seen from the Earth are the source apparent angular diameters. For simplicity the arcs are centered at the sources.

\section{Discussion}

Figure 3 shows that the average apparent angular diameters obtained increase fast as frequency decreases. The value of $D=180^{\circ}$ expected at the Earth extrapolates very well the rest of the data. The fact that the diameters are unexpectedly large can be seen in Figure 4. Here we show for comparison a curve representing the angles subtended at the Earth by a heliocentric sphere of radius $r$, that is, the largest angular diameter compatible with the assumptions of a spherical electron density distribution and absence of radiation scattering. The circle corresponds to the expected value at the Earth. Crosses represent average values while the ends 


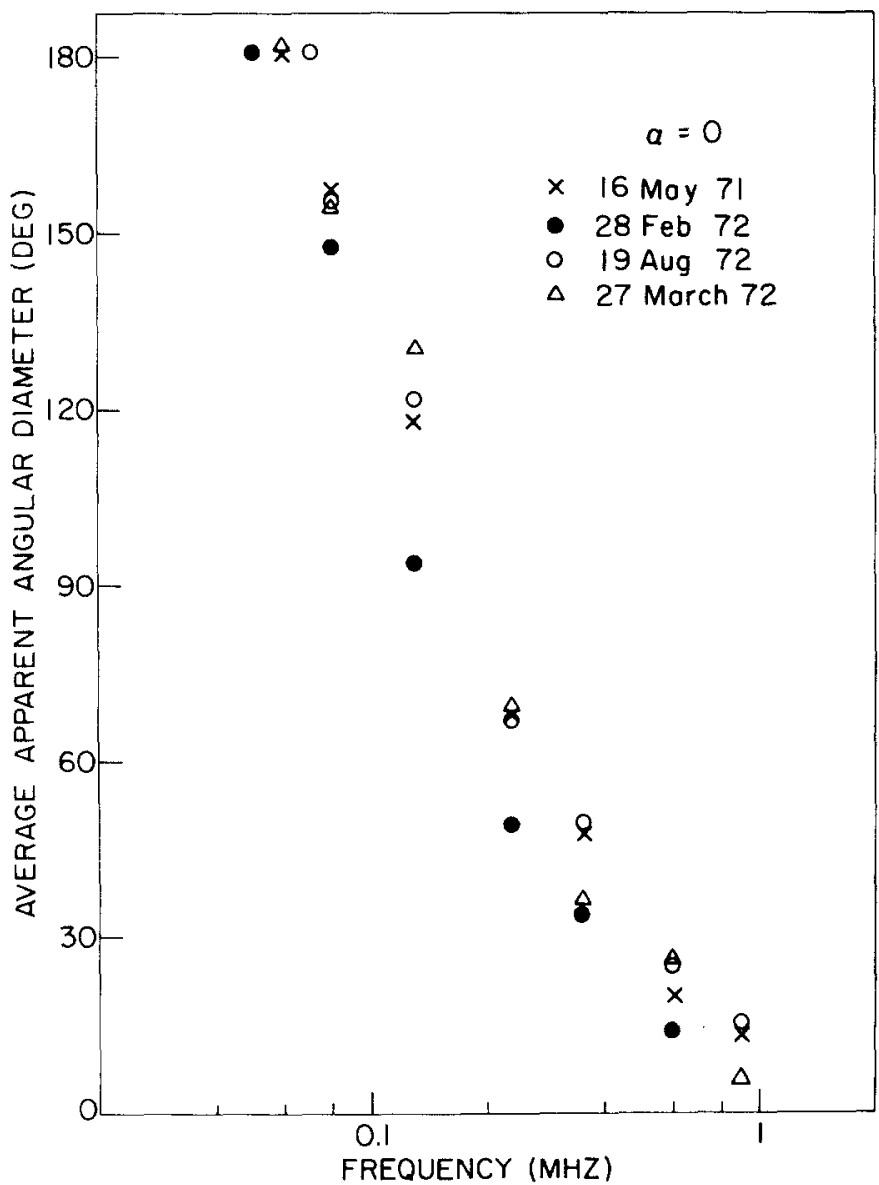

Fig. 3. Apparent angular diameter averaged during the burst lifetime. It is assumed that the sources are in the plane of rotation of the dipole $(\alpha=0)$. The points at $D=180^{\circ}$ were not observed but they were predicted when the source is at the Earth.

of the vertical bars represent the extreme values. We observe that the measurements may be larger by up to a factor of 2 . If we assume any model in which the radiation is concentrated towards the center of the source the derived diameters will be larger than those obtained here. We do not know to what extent these large apparent angular sizes can be accounted for by radiation scattering. This will have to wait until new theoretical scattering computations are available at low frequencies. Figure 5 illustrates a different aspect of the same problem. It can be seen that the tangent drawn from the Earth to the western side of a plasma level is close to the corresponding source direction. Assuming that the angle measuring the source apparent size is bisected by the Earth-source direction, it is seen that the western edge of the sources extends far outside the plasma levels. This occurs at all frequencies and it is especially noticeable in the $80 \mathrm{kHz}$ burst $(40 \mathrm{kHz}$ plasma level). 


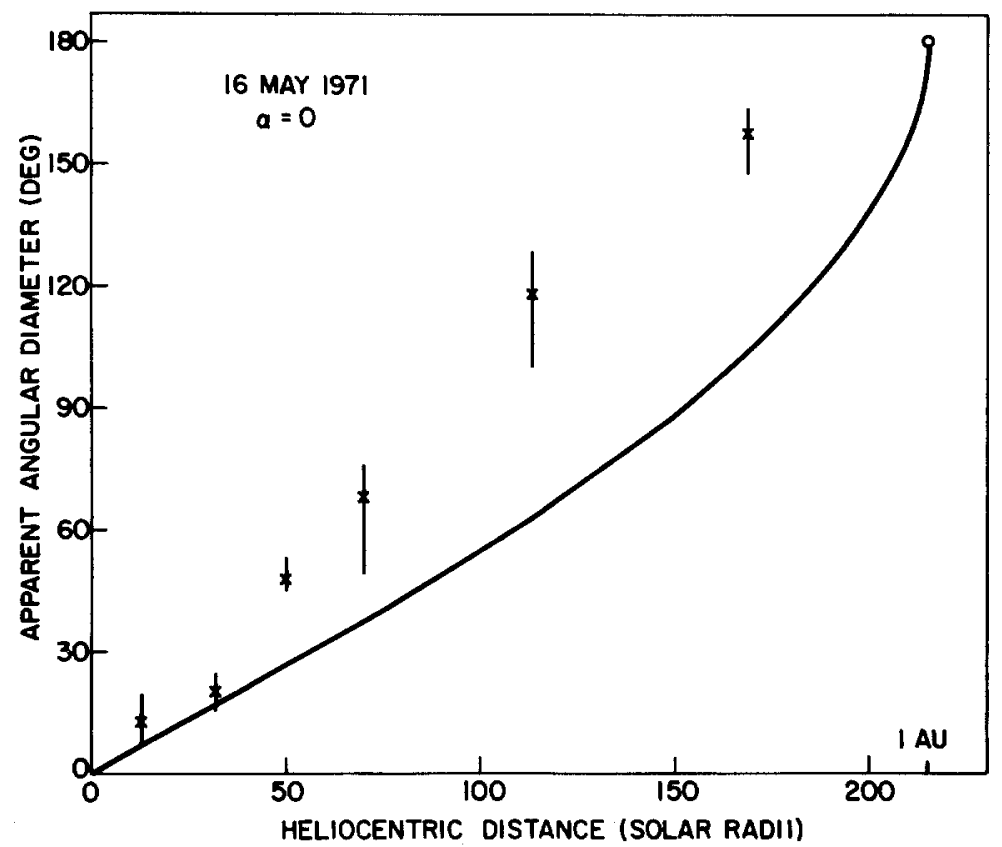

Fig. 4. Source apparent angular diameter as a function of heliocentric distance. For the distance scale see the text. The $x$ 's represent average values. The ends of the verticle bars are the extreme values measured during the burst lifetime. The curve represents the angle subtended at the Earth by a sphere concentric with the Sun. The circle at $D=180^{\circ}$ is the value predicted when the source reaches the Earth.

Kellogg (1975) has reported similarly large sizes for frequencies between 126 and $200 \mathrm{kHz}$. Besides, his measurements of one event indicate that the angular distances of the sources from the plane of the ecliptic, as seen from the Earth, were not more than $15^{\circ}$. Kellogg's results do not contradict the $\alpha=0$ assumption. large sizes have been reported also by Baumback et al. (1975).

We have considered other reasons to account for the large apparent source sizes: (1) The receiver bandwidth is $10 \mathrm{kHz}$ centered on the observing frequencies. The low frequency end of the band at $80 \mathrm{kHz}$, for example, receives second harmonic radiation from a plasma level of $37.5 \mathrm{kHz}$. This is about only $10 R_{\odot}$ further out than the $40 \mathrm{kHz}$ plasma level and could not contribute appreciably to the source apparent size. We conclude that the bandwidth effect is negligible. (2) The galactic background is a $4 \pi$ stereoradian source and tends to produce a lower parameter $C$. Because of the relative strength of the galactic background and of the radio bursts, we have estimated that the effect is negligible at and below $900 \mathrm{kHz}$. The galactic background is strong at this frequency. (3) Simultaneous presence of fundamental radiation from plasma level $f_{0}$ and second harmonic radiation from plasma level $f_{\mathrm{o}} / 2$. This seems unlikely but we do not have measurements accurate enough to rule it out completely. For trajectories that pass near the Earth the angular separation of fundamental and second harmonic sources is small compared to the measured apparent sizes. (4) Earth noise 


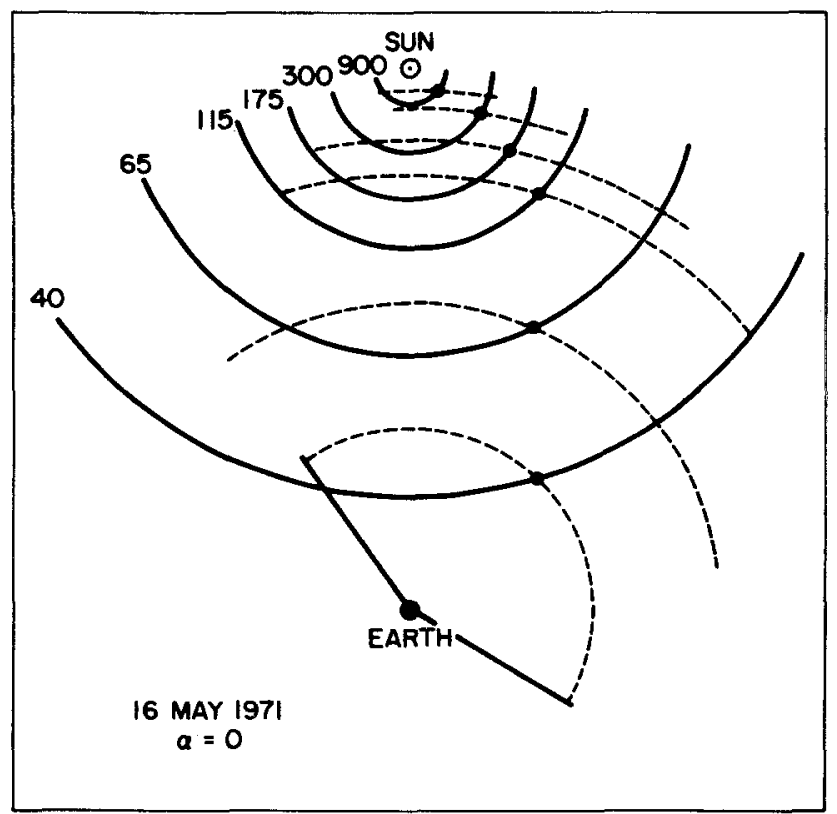

Fig. 5. Dots represent the source positions and solid circles represent plasma levels. Broken line ares define the source apparent angular extent, as seen from the Earth. The sources are assumed at the center of the broken line arcs. It is seen that in this model the radiation seems to be coming in from regions far outside the plasma levels, at all frequencies. Numbers are plasma frequencies in $\mathrm{kHz}$. The radiation observed below $900 \mathrm{kHz}$ was second harmonic.

contamination. At the time of the 16 May, 1971 burst IMP-6 was about $200000 \mathrm{~km}$ from the Earth, on the day side. There is no indication on our records of Earth activity that would appreciably modify the results. (5) Reflection of source rays by the Earth. We are in the process of studying this effect. (6) Inclination of the spin plane with respect to the solar equator. For simplicity it has been assumed that the sources are on the ecliptic. A more realistic model probably is to assume that they are on the solar equator plane. However, for practical purposes the spin plane is on the plane of the ecliptic and the $7^{\circ}$ inclination of the solar equator has an undetectable effect.

The $\alpha=0$ assumption has been adopted for the following reasons. First, excited electrons were observed at the Earth for these events. Second, it is the simplest assumption. Third, except at the low frequencies, large $\alpha$ 's imply large distances from the source to the Sun. Further, we have done some preliminary work with models using three dimensional trajectories, assuming point sources and neglecting radio wave scattering. We find that to explain the observed apparent sizes the sources must be far from the ecliptic at positions that imply heliocentric distances too large to be compatible with the electron density distribution model used. We have seen that at distances larger than about $\frac{1}{3}$ or $\frac{1}{2} \mathrm{AU}$ the model does not contradict Kellogg's observations. Closer to the Sun eclipse observations show how the Sun's polar field extends to lower heliographic latitudes with increasing distance (Schatten, 1972). 
In connection with Figure 4, it was mentioned that we noticed a systematic change of the modulation parameter during the life time of some bursts. In terms of the $\alpha=0$ assumption the variation can be interpreted as an increase in apparent angular size with time. There seems to be two different rates of increase, one for the rising part of the burst to the peak, the other for the decaying part. The increase is faster during the rising part where the measurements are also less reliable. In a first approximation, the apparent angular size in the post-maximum region seems to increase at a constant rate. There are indications that this rate decreases with decreasing frequency. There is little doubt that the apparent increase is real. The only reference we have found in the literature about a similar effect has been given by Weiss and Sheridan (1962) who, observing type III bursts at 40 and $60 \mathrm{MHz}$, reported an undoubtful increase in the angular sizes during the burst lifetime. Based on scattering analysis at meter wavelength Riddle (1974) predicted a source broadening that may be related to the effect reported here.

\section{Summary}

We have presented preliminary results on the apparent angular sizes of the type III sources observed at hectometric and kilometric wavelength. The observations were obtained with a short dipole rotating in the plane of the ecliptic where the sources were assumed to be. The results show that the apparent angular sizes are unexpectedly large, and that they increase fast with decreasing frequency. After discussing several reasons to explain the large angular sizes of these events, it seems that scattering of radio waves in the interplaneatary medium is the most likely cause. The altitude of the sources above the ecliptic plane may sometimes play a role, but a preliminary analysis indicates that it may be of secondary importance for these events.

We have found also that the apparent angular sizes increase with time during the life of the burst.

We are in the process of studying the data in more detail. We expect that some of the answer to the problems posed by our observations will be found when more scattering computations for low frequencies become available.

\section{Acknowledgements}

I would like to acknowledge the support of Prof. F. T. Haddock. Mr William H. Potter wrote the computer program to do the amplitude modulation analysis, entertained many useful discussions and critically read a draft. This work was done under NASA Grant NSG-7004.

\section{References}

Alvarez, H., Lin, R. P., and Bame, S. J.: 1975, Solar Phys. 44, 485.

Baumback, M., Kurth, W., and Gurnett, D. A.: 1975, these Proceedings, p. 475.

Elgaroy, O. and Rodberg, H.: 1963, Astrophys. Norv. 8, $2 \pi 1$. 
Goldstein, S. J.: 1959, Astrophys. J. 130, 393.

Kellogg, P.: 1975, these Proceedings, p. 449.

Riddle, A.: 1974, Solar Phys. 35, 153.

Schatten, K. H.: 1972, in C. P. Sonnet, P. J. Coleman, Jr, and J. M. Wilcox (eds.), Solar Wind, p. 65. Steinberg, J. L.: 1972, Astron. Astrophys. 18, 382.

Weiss, A. A., and Sheridan, K. V.: 1962, J. Phys. Soc. Japan 17, Suppl. A-11, 223.

Wild, J. P., and Sheridan, K. V.: 1958, Proc. IRE 46, 160. 